\title{
STRONGLY SEPARABLE PAIRINGS OF RINGS
}

\author{
BY \\ ROBERT S. CUNNINGHAM( $\left.{ }^{1}\right)$
}

\begin{abstract}
The theory of adjoint functors has been used by Morita to develop a theory of Frobenius and quasi-Frobenius extensions subsuming the work of Kasch, Müller, Nakayama, and others. We use adjoint functors to define a pairing of the two rings and develop a theory of relative projective and injective modules for pairings generalizing that of Hochschild for extensions.

The main purpose of this paper is to define "strongly separable pairings" generalizing strongly separable (i.e. finitely generated projective separable) algebras. We show that such pairings have very close connections to category equivalences, so that it is natural to investigate those properties shared by two rings which admit a strongly separable pairing. We show that most "categorical" properties are so shared.
\end{abstract}

Introduction. Throughout this paper we shall assume that all rings are associative and have an identity, and that all modules, subrings, and extensions are unital. We shall further assume that all functors between categories of modules are covariant and additive.

Frequently in ring theory we find two rings, $R$ and $S$, that are related in some specific way. From our knowledge of this relation we wish to study the structure of one of the rings in terms of the other. Familiar examples include

(1) algebras over a commutative ring,

(2) general extensions of a given ring,

(3) endomorphism rings of finitely generated projective modules.

In each of these examples the relation determines what we shall call an "adjoint triple" of functors between the categories ${ }_{R} \mathscr{M}$ and ${ }_{s} \mathscr{M}$ of left modules over $R$ and $S$. As Morita observed in [20], much of the relative structure of $R$ and $S$ is determined by this adjoint triple.

Let $R$ and $S$ be rings. For covariant additive functors $F:{ }_{R} \mathscr{M} \rightarrow{ }_{S} \mathscr{M}$ and $G:{ }_{s} \mathscr{M} \rightarrow{ }_{R} \mathscr{M}$, we say that $G$ is a left adjoint of $F$ and that $F$ is a right adjoint of $G$ in case there is a natural isomorphism

$$
\operatorname{Hom}_{R}(G(N), M) \cong \operatorname{Hom}_{S}(N, F(M))
$$

Received by the editors September 2, 1969.

AMS Subject Classifications. Primary 1690, 1720.

Key Words and Phrases. Pairings of rings, adjoint functors, separable algebras, relative homological algebra, quasi-Frobenius extensions, Frobenius extensions, category equivalences.

( $\left.{ }^{1}\right)$ This paper is taken from the author's doctoral dissertation written under the direction of F. W. Anderson at the University of Oregon. This work was partially supported by a NSF Traineeship at the University of Oregon.

The author wishes to express his appreciation to Professor Anderson for his guidance and encouragement.

Copyright (C) 1970, American Mathematical Society 
for $M \in_{R} \mathscr{M}$ and $N \in{ }_{s} \mathscr{M}$. If $G$ is a left adjoint of $F$ and $F$ has a right adjoint $H:{ }_{s} \mathscr{M} \rightarrow{ }_{R} \mathscr{M}$, we shall say that the triple $(G, F, H)$ is an adjoint triple. Such an adjoint triple of functors is uniquely determined up to natural isomorphism by any one of the functors $G, F$, or $H$.

For two rings $R$ and $S$, we define a pairing of $R$ and $S$ to be an adjoint triple $(G, F, H)$ where $F:{ }_{R} \mathscr{M} \rightarrow{ }_{s} \mathscr{M}$ and $G, H:{ }_{s} \mathscr{M} \rightarrow{ }_{R} \mathscr{M}$. We shall say that $R$ and $S$ are paired if there exists such a pairing.

In $\S 1$ we review Morita's characterization of adjoint triples of functors, and his generalization [20] of the Frobenius and quasi-Frobenius extensions of Kasch [18], Müller [22], [23], and others.

In $\$ 2$ we use methods of Morita [20] to develop a theory of relative projective and injective modules for pairings generalizing that of Hochschild [14] for extensions.

Pairings may be used naturally to study the separable algebras of Auslander and Goldman [2]. In \$3 we define a "strongly separable pairing” generalizing strongly separable (i.e. finitely generated projective separable) algebras and give the basic properties of such pairings. In $\$ 4$ we then use a pairing associated with ring extensions to define "strongly separable extensions" of rings and generalize certain theorems of Auslander and Goldman to these extensions.

We show in $\$ 3$ that strongly separable pairings are closely connected with the theory of category equivalences. It is natural to ask what properties are shared by rings which are strongly separably paired. In $\$ 5$ we show that most properties shared by rings with equivalent categories of modules are also shared by strongly separably paired rings. Some examples are left and right homological dimensions and chain conditions on left or right ideals. However, the lattices of two-sided ideals of such rings need not be isomorphic.

1. Adjoint triples of functors. Let $F:{ }_{R} \mathscr{M} \rightarrow{ }_{s} \mathscr{M}$ and $G, H:{ }_{s} \mathscr{M} \rightarrow{ }_{R} \mathscr{M}$. In case $G$ is a left adjoint of $F$ and $H$ is a right adjoint of $F$, that is, in case there are natural isomorphisms

$$
\begin{aligned}
& \eta_{N, M}: \operatorname{Hom}_{R}(G(N), M) \rightarrow \operatorname{Hom}_{S}(N, F(M)), \\
& \lambda_{M, N}: \operatorname{Hom}_{S}(F(M), N) \rightarrow \operatorname{Hom}_{R}(M, H(N))
\end{aligned}
$$

for all $M \in_{R} \mathscr{M}$ and $N \in_{S} \mathscr{M}$, we shall say that the triple $(G, F, H)$ is an adjoint triple of functors. By a theorem of $\operatorname{Kan}$ [17], an adjoint triple $(G, F, H)$ is determined to within natural isomorphism by $F$. Moreover, in [20] Morita gave the following characterization of adjoint triples.

1.1. TheOREM. Let $(G, F, H)$ be a triple of functors with $F:{ }_{R} \mathscr{M} \rightarrow{ }_{S} \mathscr{M}$ and $G, H:{ }_{s} \mathscr{M} \rightarrow{ }_{R} \mathscr{M}$. Then $(G, F, H)$ is an adjoint triple iff for $M \in_{R} \mathscr{M}$ and $N \in{ }_{s} \mathscr{M}$ there are natural isomorphisms

$$
F(M) \cong{ }_{S} P_{R} \otimes_{R} M, \quad G(N) \cong{ }_{R} Q_{S} \otimes_{S} N, \quad H(N) \cong{ }_{R} \operatorname{Hom}_{S}\left({ }_{S} P_{R, S} N\right)
$$


with bimodules ${ }_{S} P_{R}$ and ${ }_{R} Q_{S}$ satisfying either

(a) $P_{R}$ is finitely generated and projective,

(b) ${ }_{R} Q_{S} \cong{ }_{R} P_{S}^{*}={ }_{R} \operatorname{Hom}_{R}\left({ }_{S} P_{R}, R\right)_{S}$

or, equivalently,

$\left(\mathrm{a}^{\prime}\right)_{R} Q$ is finitely generated and projective,

$\left(\mathrm{b}^{\prime}\right){ }_{S} P_{R} \cong{ }_{S} Q_{R}^{*}={ }_{S} \mathrm{Hom}_{R}\left({ }_{R} Q_{S}, R\right)_{R}$.

Specifically, if (a) and (b) hold, then for $p \in P, m \in M$, and $n \in N$, natural isomorphisms

$\lambda_{M, N}: \operatorname{Hom}_{S}(F(M), N) \rightarrow \operatorname{Hom}_{R}(M, H(N))$ and

$\eta_{N, M}: \operatorname{Hom}_{R}(G(N), M) \rightarrow \operatorname{Hom}_{S}(N, F(M))$

are given by

$$
\left[\lambda_{M, N}(\psi)(m)\right](p)=\psi(p \otimes m) \text { for } \psi \in \operatorname{Hom}_{S}(F(M), N)
$$

and

$$
\eta_{N, M}(\varphi)(n)=\sum_{\alpha} x_{\alpha} \otimes \varphi\left(f_{\alpha} \otimes n\right) \quad \text { for } \varphi \in \operatorname{Hom}_{R}(G(N), M)
$$

where $\left\{x_{\alpha}\right\}$ and $\left\{f_{\alpha}\right\}$ are a dual basis for $P_{R}$.

When we wish to emphasize the bimodule ${ }_{S} P_{R}$ which corresponds to an adjoint triple $(G, F, H)$, we shall call $P$ the bimodule associated with the adjoint triple. As a consequence of Theorem 1.1, there is a one-to-one correspondence betwen pairings of $R$ and $S$ and isomorphism classes of bimodules ${ }_{S} P_{R}$ which are finitely generated and projective as $R$-modules.

1.2. EXAMPLE. Suppose there is a unital ring homomorphism $R \rightarrow S$ (e.g., $R$ is a subring of $S$, or $R$ is commutative and $S$ is an $R$-algebra). Then ${ }_{R} S_{S}$ is a bimodule and $S_{S}$ is finitely generated and projective, so the functors

$$
\begin{array}{ll}
H:{ }_{S} \mathscr{M} \rightarrow{ }_{R} \mathscr{M} & \text { via } H(N)={ }_{R} S_{S} \otimes_{S} N \cong{ }_{R} N, \\
F:{ }_{R} \mathscr{M} \rightarrow{ }_{S} \mathscr{M} & \text { via } F(M)={ }_{S} S_{R} \otimes_{R} M \cong \operatorname{Hom}_{S}\left({ }_{R} S_{S}, S\right) \otimes_{R} M, \\
E:{ }_{R} \mathscr{M} \rightarrow{ }_{S} \mathscr{M} & \text { via } E(M)={ }_{S} \operatorname{Hom}_{R}\left({ }_{R} S_{S},{ }_{R} M\right)
\end{array}
$$

form a pairing $(F, H, E)$ of $S$ and $R$. If further $S_{R}$ is finitely generated and projective, the functor

$$
G:{ }_{S} \mathscr{M} \rightarrow{ }_{R} \mathscr{M} \quad \text { via } G(N)=\operatorname{Hom}_{R}\left({ }_{S} S_{R}, R\right) \otimes_{S} N={ }_{R} S_{S}^{*} \otimes_{S} N
$$

gives a pairing $(G, F, H)$ of $R$ and $S$.

Let $R$ and $S$ be rings. A bimodule ${ }_{S} N_{R}$ is similar to a bimodule ${ }_{S} N_{R}^{\prime}$ if there are bimodule homomorphisms

and

$$
\alpha: N \rightarrow \oplus \sum_{n} N^{\prime} ; \quad \alpha^{\prime}: \oplus \sum_{n} N^{\prime} \rightarrow N
$$

$$
\beta: N^{\prime} \rightarrow \oplus \sum_{m} N ; \quad \beta^{\prime}: \oplus \sum_{m} N \rightarrow N^{\prime}
$$

such that $\alpha^{\prime} \alpha=1_{N}$ and $\beta^{\prime} \beta=1_{N^{\prime}}$. 
In this case, we write ${ }_{S} N_{R} \sim_{S} N_{R}^{\prime}$. Similarity is likewise defined for one-sided modules.

Analogously, for functors $G, H:{ }_{R} \mathscr{M} \rightarrow{ }_{S} \mathscr{M}$, we shall say that $G$ is similar to $H$ if there are natural transformations

$$
\varphi_{M}: G(M) \rightarrow \oplus \sum_{n} H(M) ; \quad \varphi_{M}^{\prime}: \oplus \sum_{n} H(M) \rightarrow G(M)
$$

and

$$
\theta_{M}: H(M) \rightarrow \oplus \sum_{m} G(M) ; \quad \theta_{M}^{\prime}: \oplus \sum_{m} G(M) \rightarrow H(M)
$$

such that $\varphi_{M}^{\prime} \varphi_{M}=1_{G(M)}$ and $\theta_{M}^{\prime} \theta_{M}=1_{H(M)}$.

Again in this case we write $G \sim H$. If $G(M)={ }_{S} N_{R} \otimes_{R} M$ and $H(M)={ }_{S} N_{R}^{\prime} \otimes_{R} M$, it is clear that $G \sim H$ iff ${ }_{S} N_{R} \sim{ }_{S} N_{R}^{\prime}$.

Let $(G, F, H)$ be a pairing of $R$ and $S$, and consider the following conditions on $G$ and $H$.

(1) $G$ and $H$ are naturally equivalent.

(2) $G \sim H$.

If (1) holds, we shall say that $(G, F, H)$ is a Frobenius pairing of $R$ and $S$, while we shall call $(G, F, H)$ a quasi-Frobenius (QF) pairing of $R$ and $S$ in case (2) holds.

1.3. TheOREM [20, TheOREM 4.1]. Let $(G, F, H)$ be a pairing of $R$ and $S$ with associated bimodule ${ }_{S} P_{R}$. Then $(G, F, H)$ is a $Q F$ pairing iff ${ }_{S} P_{R}$ satisfies

(a) ${ }_{S} P$ and $P_{R}$ are finitely generated and projective,

(b) ${ }_{R} \operatorname{Hom}_{R}\left({ }_{S} P_{R}, R\right)_{S} \sim_{R} \operatorname{Hom}_{S}\left({ }_{S} P_{R}, S\right)_{S}$.

The pairing $(G, F, H)$ is Frobenius iff ${ }_{S} P_{R}$ satisfies (a) and

$\left(\mathrm{b}^{\prime}\right)_{R} \operatorname{Hom}_{R}\left({ }_{S} P_{R}, R\right)_{S} \cong{ }_{R} \operatorname{Hom}_{S}\left({ }_{S} P_{R}, S\right)_{S}$.

If $(G, F, H)$ is a $\mathrm{QF}$ pairing, the functor $G$ must have a left adjoint since $G \sim H$. By uniqueness of adjoints, this functor must be similar to $F$. We thus have the following additional characterizations of Frobenius and QF pairings.

1.4. Corollary. Let $(G, F, H)$ be a pairing of $R$ and $S$ with associated bimodule ${ }_{S} P_{R}$. Then $(G, F, H)$ is a $Q F$ pairing iff ${ }_{S} P_{R}$ satisfies

(a) ${ }_{S} P$ and $P_{R}$ are finitely generated and projective,

(b) ${ }_{S} P_{R} \sim{ }_{S} \mathrm{Hom}_{S}\left({ }_{R} P_{S}^{*}, S\right)_{R}$.

The pairing $(G, F, H)$ is Frobenius iff ${ }_{S} P_{R}$ satisfies (a) and

(b') ${ }_{S} P_{R} \cong{ }_{S} \operatorname{Hom}_{S}\left({ }_{R} P_{S}^{*}, S\right)_{R}$.

Let $S$ be an extension of $R$ with $S_{R}$ finitely generated and projective, and recall the pairing $(G, F, H)$ of Example 1.2. Following Morita, we say that $S$ is a Frobenius extension of $R$ in case this pairing is Frobenius, and that $S$ is a quasiFrobenius (QF) extension of $R$ in case this pairing is QF. As Morita showed, these concepts generalize the Frobenius and QF extensions studied by Kasch, Müller, Nakayama, and others. By Theorem $1.3, S$ is a QF extension of $R$ iff ${ }_{R} S_{S} \sim_{R} \operatorname{Hom}_{R}\left({ }_{S} S_{R}, R\right)_{S}$, while $S$ is a Frobenius extension of $R$ iff

$$
{ }_{R} S_{S} \cong{ }_{R} \operatorname{Hom}_{R}\left({ }_{S} S_{R}, R\right)_{S}
$$


2. Relative projective and injective modules for pairings. Let $R$ and $S$ be rings and let $F:{ }_{R} \mathscr{M} \rightarrow{ }_{s} \mathscr{M}$ be a functor. For $M \in{ }_{R} \mathscr{M}$, we say $M$ is $F$-projective in case for any diagram of $R$-modules

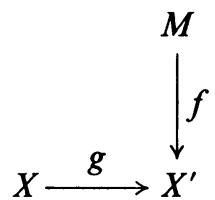

such that $F(g): F(X) \rightarrow F\left(X^{\prime}\right)$ is a split epimorphism, there is an $R$-homomorphism $h: M \rightarrow X$ such that $f=g h$. Similarly, we say that $M$ is $F$-injective in case for any diagram of $R$-modules

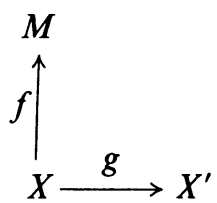

such that $F(g): F(X) \rightarrow F\left(X^{\prime}\right)$ is a split monomorphism, there is an $R$-homomorphism $h: X^{\prime} \rightarrow M$ such that $f=h g$.

These definitions generalize those of Hochschild [14], for if $S$ is an extension of $R$ and $H:{ }_{s} \mathscr{M} \rightarrow{ }_{R} \mathscr{M}$ is the restriction functor of Example 1.2, the $H$-projective and $H$-injective $S$-modules are precisely the $(S, R)$-projective and $(S, R)$-injective $S$-modules respectively.

It is clear from our definitions that direct summands and finite direct sums of $F$-projective or $F$-injective modules are again $F$-projective or $F$-injective.

In our development below we characterize relative projective and injective modules for functors in adjoint triples. We do not, in general, require the full strength of such triples for our results. Those characterizations of relative projective modules require only that our functor have a left adjoint, while those of relative injective modules require only that our functor have a right adjoint.

2.1. Proposition (MORITA). Let $(G, F, H)$ be a pairing of $R$ and $S$ with associated bimodule ${ }_{S} P_{R}$. Then for $M \in{ }_{R} \mathscr{M}$ and $N \in{ }_{S} \mathscr{M}$, there are natural transformations

defined via

$$
\begin{aligned}
\alpha_{N}: N & \rightarrow F G(N), & \alpha_{N}^{\prime}: F H(N) & \rightarrow N, \\
\beta_{M}: M & \rightarrow H F(M), & \beta_{M}^{\prime}: G F(M) & \rightarrow M
\end{aligned}
$$

$$
\begin{array}{rlrl}
\alpha_{N} & =\eta_{N, G(N)}\left(1_{G(N)}\right), & & \alpha_{N}^{\prime}=\lambda_{H(N), N}^{-1}\left(1_{H(N)}\right) \\
\beta_{M}=\lambda_{M, F(M)}\left(1_{F(M)}\right), & & \beta_{M}^{\prime}=\eta_{F(M), M}^{-1}\left(1_{F(M)}\right)
\end{array}
$$

for $\eta, \lambda$ as in Theorem 1.1. Let $\left\{x_{i}\right\},\left\{f_{i}\right\}$ be a dual basis for $P_{R}$. Then for $n \in N, m \in M$, and $p \in P$,

$$
\begin{aligned}
\alpha_{N}(n) & =\sum_{i} x_{i} \otimes f_{i} \otimes n, \\
\alpha_{N}^{\prime}(p \otimes h) & =h(p) \text { for } h \in H(N)=\operatorname{Hom}_{S}\left({ }_{S} P_{R, S} N\right), \\
{\left[\beta_{M}(m)\right](p) } & =p \otimes m, \\
\beta_{M}^{\prime}(g \otimes p \otimes m) & =g(p) m \text { for } g \in P^{*} .
\end{aligned}
$$


Morita [20, Lemmas $\left.6.1,6.1^{\prime}\right]$ showed that for any $M \in_{R} \mathscr{M}, F(M)$ is both $G$ injective and $H$-projective. Using methods similar to his, it is easy to see that for any $N \in{ }_{s} \mathscr{M}, G(N)$ is $F$-projective and $H(N)$ is $F$-injective. This gives us the following characterization of relative projective and injective modules.

2.2. TheOREM. Let $(G, F, H)$ be a pairing of $R$ and $S$, and let $M \in_{R} \mathscr{M}$ and $N \in{ }_{s} \mathscr{M}$. Then

(a) $M$ is F-projective iff $\beta_{M}^{\prime}: G F(M) \rightarrow M$ is a split epimorphism

(b) $M$ is F-injective iff $\beta_{M}: M \rightarrow H F(M)$ is a split monomorphism

(c) $N$ is $H$-projective iff $\alpha_{N}^{\prime}: F H(N) \rightarrow N$ is a split epimorphism

(d) $N$ is G-injective iff $\alpha_{N}: N \rightarrow F G(N)$ is a split monomorphism.

Proof. (c) and (d) are due to Morita [20, 6.3, 6.3']; (a) and (b) are similar.

Although our definitions of relative projective and injective modules extend those of Hochschild, they bear little resemblance to his. In fact, Theorem 2.2 (a) shows that if $P_{R}$ is not a generator, projective modules need not be $F$-projective. For want of better terms, we shall call an $R$-module $M$ weakly $F$-projective in case for any exact sequence $0 \rightarrow M^{\prime} \rightarrow M^{\prime \prime} \rightarrow M \rightarrow 0$ of $R$-modules, the sequence splits in ${ }_{R} \mathscr{M}$ iff the sequence $0 \rightarrow F\left(M^{\prime}\right) \rightarrow F\left(M^{\prime \prime}\right) \rightarrow F(M) \rightarrow 0$ is split exact in ${ }_{s} \mathscr{M}$, while we shall say that $M$ is weakly $F$-injective in case for any exact sequence $0 \rightarrow M \rightarrow M^{\prime} \rightarrow M^{\prime \prime} \rightarrow 0$ of $R$-modules, the sequence splits in ${ }_{R} \mathscr{M}$ iff the sequence $0 \rightarrow F(M) \rightarrow F\left(M^{\prime}\right) \rightarrow F\left(M^{\prime \prime}\right) \rightarrow 0$ is split exact in ${ }_{R} \mathscr{M}$.

Clearly projective and injective $R$-modules are weakly $F$-projective and weakly $F$-injective respectively. Further, $F$-projective modules are weakly $F$-projective, for consider the diagram

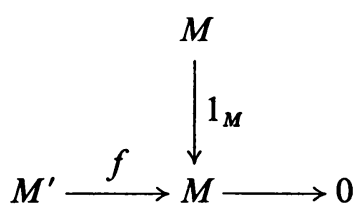

and assume $M$ is $F$-projective. If $F\left(M^{\prime}\right) \rightarrow F(M) \rightarrow 0$ is split exact, then the definition of $F$-projective modules gives us a map $g: M \rightarrow M^{\prime}$ splitting $f$, and $M$ is weakly $F$-projective. Similarly, $F$-injective modules are weakly $F$-injective. The following theorem gives necessary and sufficient conditions for weakly $F$-projective and weakly $F$-injective modules to coincide with the $F$-projective and $F$-injective modules.

2.3. THEOREM. Let $(G, F, H)$ be a pairing of $R$ and $S$ with associated bimodule ${ }_{S} P_{R}$. Then the following are equivalent:

(a) every weakly F-projective ${ }_{R} M$ is F-projective,

(b) every projective ${ }_{R} M$ is F-projective, 
(c) every weakly $F$-injective ${ }_{R} M$ is $F$-injective,

(d) every injective ${ }_{R} M$ is $F$-injective,

(e) $P_{R}$ is a progenerator.

Proof. (a) implies (b) and (c) implies (d) are clear.

(b) implies (e). If every projective module is $F$-projective, then ${ }_{R} R$ is $F$-projective. So by Theorem 2.2, $\beta_{R}^{\prime}: G F(R) \rightarrow R$ is an epimorphism. But then there are $f_{i} \in P^{*}$ and $p_{i} \in P$ with $\sum_{i} f_{i}\left(p_{i}\right)=1_{R}$, so that $P_{R}$ is a generator [20].

(d) implies (e). Suppose every injective ${ }_{R} M$ is $F$-injective. Let $0 \neq_{R} A$ be arbitrary; we claim that $P \otimes_{R} A \neq 0$. Let ${ }_{R} E$ be the injective hull of $A$. Since $E$ is $F$-injective, $\beta_{E}: E \rightarrow H F(E)$ is a monomorphism, so $\beta_{E}(x) \neq 0$ for all $0 \neq x \in E$. But $\beta_{E}$ is defined by $\beta_{E}(x)(p)=p \otimes x$; thus for any $0 \neq x \in E, P \otimes_{R} R x \neq 0$. In particular, $P \otimes_{R} R x \neq 0$ for any $0 \neq x \in A$, so that $P \otimes_{R} A \neq 0$. Thus by [15, Theorem 2.1], $P_{R}$ is faithfully flat. It follows from [15, Propositions 2.3 and 2.4] that $P_{R}$ is a generator.

(e) implies (a). Suppose that $P_{R}$ is a generator. Then there are $f_{i} \in P^{*}$ and $p_{i} \in P$ such that $\sum_{i} f_{i}\left(p_{i}\right)=1_{R}$. Thus for any $M \in{ }_{R} \mathscr{M}$, and any $m \in M$, we have

$$
\beta_{M}^{\prime}\left(\sum_{i} f_{i} \otimes p_{i} \otimes m\right)=\sum_{i} f_{i}\left(p_{i}\right) m=m,
$$

so $\beta_{M}^{\prime}$ is an epimorphism.

Suppose that $M$ is weakly $F$-projective. Since $\beta_{M}^{\prime}: G F(M) \rightarrow M$ is an epimorphism, and since $F\left(\beta_{M}^{\prime}\right) \alpha_{F(M)}=1_{F(M)}$ by [20, (53)], $F\left(\beta_{M}^{\prime}\right): F G F(M) \rightarrow F(M)$ is a split epimorphism. Thus since $M$ is weakly $F$-projective, we must have $\beta_{M}^{\prime}$ split; i.e., $M$ must be $F$-projective.

(e) implies (c). This will follow by an argument similar to that of (e) implies (a) once we show that $\beta_{M}: M \rightarrow H F(M)$ is a monomorphism for all ${ }_{R} M$. But suppose $P_{R}$ is a generator and take any $M \in_{R} \mathscr{M}$ and any $m \in M$. Then if $\beta_{M}(m)=0, P \otimes_{R} R m$ $=0$, so that $R m=0$ and $m=0$. So $\beta_{M}$ is a monomorphism for any ${ }_{R} M$.

Relative homological algebra of QF extensions has been studied in [10] and [12]. It was shown there that if $S$ is a QF extension of $R$, then a module ${ }_{s} N$ is $(S, R)$-projective iff it is $(S, R)$-injective. This generalizes easily to QF pairings.

2.4. Proposition. Let $(G, F, H)$ be a $Q F$ pairing of $R$ and $S$. Then $M \in_{R} \mathscr{M}$ is F-projective iff it is F-injective.

Proof. Let $M$ be $F$-projective. Then $M$ is isomorphic to a direct summand of $G F(M)$. But $G \sim H$, so $G F(M) \sim H F(M)$; so since $H F(M)$ is $F$-injective, $M$ is $F$-injective. The converse is similar.

This property characterizes those algebras $A$ over a commutative subring $K$ such that $A$ is a QF extension of $K$.

2.5. Proposition. Let $A$ be an algebra over a subring $K$ of the center of $A$. Then - $A$ is a $Q F$ extension of $K$ iff $A_{K}$ is finitely generated and projective and the $(A, K)-$ projective and $(A, K)$-injective modules coincide. 
Proof. Assume that $A_{K}$ is finitely generated and projective and that the $(A, K)$ projective and $(A, K)$-injective modules coincide.

Since ${ }_{A} A$ is projective, it is $(A, K)$-projective and thus $(A, K)$-injective. Thus $A$ is isomorphic to a direct summand of $\operatorname{Hom}_{K}\left({ }_{K} A,{ }_{K} A\right)$ [14]. But ${ }_{K} A$ is finitely generated and projective, so there is a split epimorphism $\oplus \sum_{n} K{ }_{K} \rightarrow{ }_{K} A \rightarrow 0$. Thus there is a split epimorphism $\oplus \sum_{n}{ }_{A} \operatorname{Hom}_{K}\left({ }_{K} A, K\right) \rightarrow{ }_{A} \operatorname{Hom}_{K}\left({ }_{K} A,{ }_{K} A\right) \rightarrow 0$, so there is a split epimorphism $\oplus \sum_{n}{ }_{A} \operatorname{Hom}_{K}\left({ }_{K} A, K\right) \rightarrow{ }_{A} A \rightarrow 0$. But since $A$ is an algebra over $K$, this map is in fact a bimodule split epimorphism $\oplus \sum_{n}{ }_{A} \operatorname{Hom}_{K}\left({ }_{K} A, K\right)_{K}$ $\rightarrow{ }_{A} A_{K} \rightarrow 0$.

A similar argument, using the fact that $\operatorname{Hom}_{K}\left({ }_{K} A,{ }_{K} A\right)$ must be $(A, K)$-projective, shows that there is a bimodule split epimorphism $\oplus \sum_{m{ }_{A}} A_{K} \rightarrow{ }_{A} \operatorname{Hom}_{K}\left({ }_{K} A, K\right)_{K}$ $\rightarrow 0$. Thus ${ }_{A} A_{K} \sim{ }_{A} \operatorname{Hom}_{K}\left({ }_{K} A, K\right)_{K}$ and $A$ is a QF extension of $K$.

The converse is well known; see for example [10].

3. Strongly separable pairings. In this section we define a type of pairing generalizing strongly separable algebras over commutative rings. For this we use the theories of QF pairings and relative homological algebra developed above.

Let $A$ be an algebra over the commutative ring $K$ and consider the $(A, A)$ homomorphism $\sigma: A \otimes_{K} A \rightarrow A$ via $\sigma(x \otimes y)=x y$. Then $A$ is called a separable $K$-algebra in case $\sigma$ is $(A, A)$-split; i.e., in case there is an $(A, A)$-homomorphism $\tau: A \rightarrow A \otimes_{K} A$ with $\sigma \tau=1_{A}$. Equivalently, $A$ is separable over $K$ in case there are sets $\left\{a_{i}\right\},\left\{b_{i}\right\} \subset A$ such that

$$
\sum_{i} a_{i} b_{i}=1_{A} \text { and } a\left(\sum_{i} a_{i} \otimes b_{i}\right)=\left(\sum_{i} a_{i} \otimes b_{i}\right) a
$$

for all $a \in A$. A separable algebra is called strongly separable in case $A_{K}$ is finitely generated and projective.

The following characterization of faithful, strongly separable algebras leads to our definition of strongly separable pairings.

3.1. Proposition. Let $A$ be an algebra over a commutative subring $K$ of the center of $A$. Then $A$ is a strongly separable $K$-algebra iff

(a) $\sigma: A \otimes_{K} A \rightarrow A$ as above admits a two-sided splitting homomorphism,

(b) $A_{K}$ is a progenerator,

(c) $A$ is a $Q F$ extension of $K$.

Proof. Let $A$ be a strongly separable algebra over $K \subset A$. Then (a) holds by definition, (b) follows since $A_{K}$ is finitely generated, projective, and faithful, and (c) is by [22, Satz 44]. The converse is clear.

Recall the pairing $(G, F, H)$ associated with any finitely generated projective algebra $A$ over $K$ in Example 1.2. We may express the properties of Proposition 3.1 in terms of the functors in this pairing as follows:

(a) The map $\sigma: F H(A) \rightarrow A$ via $\sigma(a \otimes f)=f(a)$ for $a \in A, f \in H(A)$ is an $(A, A)$ split epimorphism, 
(b) the trace map $t: G F(K) \rightarrow K$ defined via $t(f \otimes a)=f(a)$ for $a \in A$, $f \in \operatorname{Hom}_{K}(A, K)$ is a split $K$-epimorphism,

(c) $(G, F, H)$ is a QF pairing.

These properties form the basis for our definition of a strongly separable pairing.

Let $(G, F, H)$ be a pairing of $R$ and $S$. We shall say that $(G, F, H)$ is a strongly separable pairing in case

(a) $\alpha_{S}^{\prime}:{ }_{S} F H(S)_{S} \rightarrow{ }_{S} S_{S}$ is an $(S, S)$-split epimorphism,

(b) $\beta_{R}^{\prime}:{ }_{R} G F(R)_{R} \rightarrow{ }_{R} R_{R}$ is an $(R, R)$-split epimorphism,

(c) $(G, F, H)$ is a $\mathrm{QF}$ pairing.

The following two propositions give useful alternative formulations of (a) and (b).

3.2. Proposi nion. Let $(G, F, H)$ be a pairing of $R$ and $S$ with associated bimodule ${ }_{S} P_{R}$. Then condition (a) is equivalent to

(a') $\alpha_{S}:{ }_{S} S_{S} \rightarrow{ }_{S} F G(S)_{S} \cong{ }_{S} \operatorname{End}_{R}\left({ }_{S} P_{R}\right)_{S}$ is an $(S, S)$-split monomorphism.

Proof. By results of [13], $\alpha_{S}^{\prime}: F H(S) \rightarrow S$ is $(S, S)$-split iff there are $\left\{p_{i}\right\} \subset P$ and $\left\{f_{i}\right\} \subset \operatorname{Hom}_{S}(P, S)$ such that $\sum_{i} f_{i}\left(p_{i}\right)=1_{S}$ and $s\left(\sum_{i} p_{i} \otimes f_{i}\right)=\left(\sum_{i} p_{i} \otimes f_{i}\right) s$ for all $s \in S$.

Suppose we satisfy (a). Define $\varphi: \operatorname{Hom}_{R}(P, P) \rightarrow S$ via $\varphi(g)=\sum_{i} f_{i}\left(g\left(p_{i}\right)\right)$ for $g \in \operatorname{Hom}_{R}(P, P)$ and $f_{i}$ and $p_{i}$ as above. Clearly $\varphi$ is a left $S$-homomorphism. But $\varphi(g)=\alpha_{S}^{\prime}\left[\left(g \otimes 1_{S}\right)\left(\sum_{i} p_{i} \otimes f_{i}\right)\right]$, so it is easy to see that $\varphi$ is an $(S, S)$-homomorphism using the above remarks.

Finally, one checks that $\varphi$ is split by $\lambda: S \rightarrow \operatorname{End}_{R}(P)$ where $\lambda(s)(p)=s p$, so that $\lambda:{ }_{S} S_{S} \rightarrow{ }_{S} F G(S)_{S}$ admits an $(S, S)$-splitting map. But $\lambda$ and $\alpha_{S}$ are the same map, so $\left(\mathrm{a}^{\prime}\right)$ follows.

Conversely, suppose that $\alpha_{S}: S \rightarrow F G(S)$ is an $(S, S)$-split monomorphism. Since $P_{R}$ is finitely generated and projective, we have ${ }_{S} F H(S)_{S}={ }_{S} P \otimes_{R} \operatorname{Hom}_{S}(P, S)_{S}$ $\cong_{S} \operatorname{Hom}_{S}\left(\operatorname{Hom}_{R}(P, P), S\right)_{S}$ by a map $\varphi$ with $[\varphi(x \otimes f)](g)=f(g(x))$ for $x \in P$, $f \in \operatorname{Hom}_{S}(P, S)$, and $g \in \operatorname{Hom}_{R}(P, P)$.

Thus any homomorphism from $\operatorname{Hom}_{R}(P, P)$ to $S$ gives an element $\sum_{i} p_{i}$ $\otimes f_{i} \in F H(S)$; an $(S, S)$-homomorphism gives this element the property that $s\left(\sum_{i} p_{i} \otimes f_{i}\right)=\left(\sum_{i} p_{i} \otimes f_{i}\right) s$ for all $s \in S$. Finally, if such a map splits $\alpha_{S}$, this element must have the property that $\sum_{i} f_{i}\left(p_{i}\right)=1_{S}$. So by our earlier remarks, $\alpha_{S}^{\prime}$ must be $(S, S)$-split.

3.3. Proposition. Let $(G, F, H)$ be a pairing of $R$ and $S$ with associated bimodule ${ }_{S} P_{R}$ such that ${ }_{S} P$ is finitely generated and projective. Then condition (b) is equivalent to

(b') $\beta_{R}:{ }_{R} R_{R} \rightarrow{ }_{R} H F(R)_{R} \cong{ }_{R} \operatorname{End}_{S}\left({ }_{S} P_{R}\right)_{R}$ is an $(R, R)$-split monomorphism.

Proof. This proof is essentially the same as that of Proposition 3.2 and is thus omitted. 
3.4. Remarks. (a) It is clear from our development that if $A$ is a strongly separable faithful $K$-algebra, the pairing $(G, F, H)$ of Example 0.3 is a strongly separable pairing.

(b) The definition of a strongly separable pairing is completely left-right and $R-S$ symmetric by conditions $\left(\mathrm{a}^{\prime}\right)$ and $\left(\mathrm{b}^{\prime}\right)$ of the propositions.

(c) Conditions (a), (b), and (c) of the definition of a strongly separable pairing imply that ${ }_{S} P$ and $P_{R}$ are progenerators, while conditions $\left(\mathrm{a}^{\prime}\right)$ and $\left(\mathrm{b}^{\prime}\right)$ of the propositions show how $S$ and $R$ sit in the endomorphism rings of $P$. This suggests the close connection between the theory of strongly separable pairings and that of category equivalences (see [4], [19]).

3.5. Proposition. Let $(G, F, H)$ be a strongly separable pairing of $R$ and $S$. Then

(a) every $M \in{ }_{R} \mathscr{M}$ is both F-projective and F-injective,

(b) every $N \in{ }_{S} \mathscr{M}$ is both $H$-projective and $H$-injective.

Proof. By Proposition 2.4, (a) follows if we show that all ${ }_{R} M$ are $F$-projective. But for any ${ }_{R} M, G F(M) \cong G F(R) \otimes_{R} M$, so since $R$ is $(R, R)$-isomorphic to a direct summand of $G F(R), M$ is isomorphic to a direct summand of $G F(M)$ and is thus $F$-projective. A similar argument gives (b).

3.6. THEOREM. Let $R$ be a commutative ring, $P_{R}$ a finitely generated projective module, and $S$ an $R$-subalgebra of $\operatorname{End}_{R}\left(P_{R}\right)$. If ${ }_{S} P_{R}$ gives a strongly separable pairing of $R$ and $S$, then $S$ is a faithful strongly separable $R$-algebra.

Proof. Since $P_{R}$ must be a progenerator, if $S r=0$ for $r \in R$, we have $S(P r)=0$; thus $\operatorname{Pr}=0$ and we must have $r=0$. Thus $S_{R}$ is faithful.

Since ${ }_{S} S$ is finitely generated and projective, and each of $P_{R},{ }_{R} P^{*}$, and $P_{S}^{*}$ must be finitely generated and projective, it follows from Propositions 5.1 and 5.6 below that ${ }_{R}\left(P \otimes_{R} P^{*} \otimes_{S} S\right)$ is finitely generated and projective. But

$$
\alpha_{S}:{ }_{S} S \rightarrow{ }_{S} P \otimes_{R} P^{*} \otimes_{S} S
$$

is an $S$-split (and hence $R$-split) homomorphism, so that ${ }_{R} S$ is isomorphic to a direct summand of ${ }_{R}\left(P \otimes_{R} P^{*} \otimes_{S} S\right)$ and so ${ }_{R} S$ is finitely generated and projective.

Finally, consider the enveloping algebra $S^{e}=S \otimes_{R} S^{0}$ of $S$ (see [2]). Since ${ }_{S} P$ and $P_{S}^{*}$ are finitely generated and projective, there is a split $S^{e}$-epimorphism $\oplus \sum S^{e} \rightarrow P \otimes_{R} P^{*}$. Further, by Proposition 3.2 there is a split $S^{e}$-epimorphism $P \otimes_{R} P^{*} \rightarrow S$. Thus $S$ is $S^{e}$-projective, so $S$ is a separable $R$-algebra.

3.7. COROLlaRY. If $S$ is strongly separably paired to the ring of integers $Z$, then $S$ is a faithful strongly separable Z-algebra.

Now let $(G, F, H)$ be a pairing of $R$ and $S$, and let $\left(G^{\prime}, F^{\prime}, H^{\prime}\right)$ be a pairing of $S$ and $T$. It follows easily from the definition of adjoint functors that $\left(G G^{\prime}, F^{\prime} F, H H^{\prime}\right)$ is a pairing of $R$ and $T$. A similar result for strongly separable pairings holds by a routine check of the definition. 
3.8. Proposition. Let $(G, F, H)$ be a strongly separable pairing of $R$ and $S$, and let $\left(G^{\prime}, F^{\prime}, H^{\prime}\right)$ be a strongly separable pairing of $S$ and $T$. Then $\left(G G^{\prime}, F^{\prime} F, H H^{\prime}\right)$ is a strongly separable pairing of $R$ and $T$.

4. Strongly separable extensions. In $\S 1$ we defined Frobenius and QF pairings to generalize Frobenius and QF extensions. This was done so that $S$ is a Frobenius (respectively QF) extension of $R$ iff the pairing $(G, F, H)$ of Example 1.2 is a Frobenius (respectively QF) pairing. We use the same technique to define strongly separable extensions of rings.

Let $S$ be an extension of $R$ with $S_{R}$ finitely generated and projective, and define functors $G, F$, and $H$ as in Example 1.2. We call $S$ a strongly separable extension of $R$ in case the pairing $(G, F, H)$ is a strongly separable pairing. For extensions, the defining conditions on such pairings become

(a) the map $\sigma: S \otimes_{R} S \rightarrow S$ via $\sigma(x \otimes y)=x y$ is an $(S, S)$-split epimorphism,

(b') ${ }_{R} R_{R}$ is an $(R, R)$-direct summand of ${ }_{R} S_{R}$,

(c) $S$ is a QF extension of $R$.

Notice that (a) is the defining condition for the separable extensions of Hirata and Sugano [13].

4.1. EXAMPLE. Let $G$ be a group with a subgroup $H$ of finite index, say $n$. If $R$ is a ring with $n R=R$, then $R G$ is a strongly separable extension of $R H$.

Proof. Hirata and Sugano [13] show that (a) is satisfied, while Kasch [18] shows that $R G$ is a Frobenius extension of $R H$. Finally, the map $R G \rightarrow R H$ via $\sum_{G} \alpha_{g} g$ $\rightarrow \sum_{H} \alpha_{g} g$ is a two-sided $R H$-homomorphism which splits the inclusion map $R H \rightarrow R G$, so we satisfy condition $\left(\mathrm{b}^{\prime}\right)$.

4.2. ExAmple. Let $R$ be any ring. Then the ring $M_{n}(R)$ of $n \times n$ matrices over $R$ is a strongly separable extension of $R$.

Proof. Again, Hirata and Sugano show that (a) is satisfied. We have ( $\left.\mathrm{b}^{\prime}\right)$ since the map $\left[r_{i j}\right] \rightarrow r_{11}$ is an $(R, R)$-homomorphism of $M_{n}(R)$ onto $R$ which is split by the inclusion map $r \rightarrow r \cdot 1_{M_{n}(R)}$ of $R$ into $M_{n}(R)$. Finally, $M_{n}(R)$ is a Frobenius extension of $R$ since $\varphi: \operatorname{Hom}_{R}\left(M_{n}(R), R\right) \rightarrow M_{n}(R)$ via $\varphi(f)=\left[f\left(e_{i j}\right)\right]^{t}$ is easily seen to be a left $M_{n}(R)$-right $R$-isomorphism.

Notice that by Theorem 3.6, if $S$ is a strongly separable extension of a commutative ring $R \subset$ center $(S)$, then $S$ is a strongly separable $R$-algebra.

We now generalize certain results of Auslander and Goldman [2] fur central separable algebras to strongly separable extensions. Recall that if $A$ is a central separable algebra over $K$, then $A^{e} \cong \operatorname{End}_{K}\left(A_{K}\right)$ as rings. We use $E=\operatorname{End}_{R}\left(S_{R}\right)$ as the analogue of the enveloping algebra in these generalizations.

4.3. Proposition. Let $S$ be a strongly separable extension of $R$, and let $E=\operatorname{End}_{R}\left(S_{R}\right)$. Then the map $\pi: E \rightarrow S$ defined via $\pi(f)=f(1)$ is a split E-epimorphism.

Proof. Clearly $\pi$ is an $E$-epimorphism. Since $S_{R}$ is a progenerator by 3.4(c), ${ }_{E} S$ is a progenerator; so ${ }_{E} S$ is projective and $\pi$ must be $E$-split. 
For the remainder of this section, let $E$ and $\pi$ be as in the above proposition. For any $E$-module $K$ and any $X \subset E$, define $\operatorname{Ann}_{K}(X)=\{k \in K \mid x k=0$ for all $x \in X\}$. If $X$ is a right ideal of $E, A n n_{K}(X)$ is clearly a left $E$-submodule of $K$.

4.4. THEOREM. Let $S$ be a strongly separable extension of $R$. Then for any ${ }_{E} M$, the map $\pi_{M}: \operatorname{Hom}_{E}(S, M) \rightarrow \mathrm{Ann}_{M}(\operatorname{ker} \pi)$ via $\pi_{M}(f)=f\left(1_{S}\right)$ is an E-isomorphism.

Proof. Since ${ }_{E} S$ is a generator, $\pi_{M}$ is an $E$-monomorphism of $\operatorname{Hom}_{E}(S, M)$ into $M$ clearly. But for $f \in \operatorname{Hom}_{E}(S, M)$, we have

$$
(\operatorname{ker} \pi) f(1)=f((\operatorname{ker} \pi) 1)=f\left((\operatorname{ker} \pi) \pi\left(1_{E}\right)\right)=f(\pi(\operatorname{ker} \pi))=f(0)=0,
$$

so that the image of $\pi_{M}$ is contained in $\mathrm{Ann}_{M}(\operatorname{ker} \pi)$.

But take any $m \in \mathrm{Ann}_{M}(\operatorname{ker} \pi)$. Then $\rho_{m}: E \rightarrow M$ via $\rho_{m}(\alpha)=\alpha m$ is in $\operatorname{Hom}_{E}(E, M)$. But ker $\rho_{m} \supset \operatorname{ker} \pi$, so $\rho_{m}$ induces a map $g \in \operatorname{Hom}_{E}(E / \operatorname{ker} \pi, M)$ $\cong \operatorname{Hom}_{E}(S, M)$ with $g(1)=\rho_{m}(1)=m$. Thus $\pi_{M}$ is onto $\operatorname{Ann}_{M}(\operatorname{ker} \pi)$.

4.5. CoRollaRY. If $S$ is a strongly separable extension of $R$, then $R=\operatorname{Ann}_{S}(\operatorname{ker} \pi)$.

Proof. Since ${ }_{E} S$ is a progenerator, $R \cong \operatorname{End}_{E}\left({ }_{E} S\right)^{0}$ via the map $r \rightarrow \rho_{r}$ by Morita [19]. Thus the inverse map $f \rightarrow f(1)$ is an isomorphism of $\operatorname{Hom}_{E}(S, S)$ onto $R$. So $R=\operatorname{Ann}_{S}(\operatorname{ker} \pi)$ by the above theorem.

5. Properties preserved by strongly separable pairings. Following the lead suggested in 3.4(c) we now investigate those "categorical" properties of rings shared by rings which are strongly separably paired.

Most of the individual properties we study are shared by rings paired somewhat more weakly than by strongly separable pairings. We shall try to give only those hypotheses we need on the pairing for each property we study. A standing corollary of these results will be their application to strongly separable pairings.

Because of the symmetries of strongly separable pairings we noted in $3.4(\mathrm{~b})$, we shall deal only with left modules and we shall only prove that if one of $R$ or $S$ has a certain property, the other must also have that property. The symmetric statements for strongly separable pairings will be a standing corollary.

We deal first with properties involving projective, injective, and flat modules.

5.1. Proposition. Let $R$ and $S$ be rings, and let ${ }_{S} P_{R}$ be a bimodule. Then

(a) ${ }_{S} P \otimes_{R} M$ is flat for all flat ${ }_{R} M$ iff ${ }_{S} P$ is flat.

If $P_{R}$ is flat, then

(b) ${ }_{S} P \otimes_{R} M$ is projective for all projective ${ }_{R} M$ iff ${ }_{S} P$ is projective. If $P_{R}$ is finitely generated and projective, then

(c) ${ }_{S} P \otimes_{R} M$ is injective for all injective ${ }_{R} M$ iff $P_{S}^{*}$ is flat.

Proof. (a) is [18, Proposition 3, p. 131], (b) follows from [1, Theorem 1.2], and (c) is [1, Theorem 2.1].

Anderson [1] called modules ${ }_{S} P_{R}$ satisfying (b) and (c) of Proposition 5.1 projectors and injectors respectively. Clearly, if ${ }_{S} P_{R}$ is the bimodule associated with a $\mathrm{QF}$ pairing, $P$ is both a projector and an injector. 
Morita [20, 6.6 and 6.6'] proved that if $(G, F, H)$ is a pairing of $R$ and $S$, then ${ }_{R} G(N)$ is projective for all projective ${ }_{S} N$ and ${ }_{R} H(N)$ is injective for all injective ${ }_{s} N$. Together with Proposition 5.1, this yields the following result.

5.2. TheOREM. Let $(G, F, H)$ be a pairing of $R$ and $S$ with associated bimodule ${ }_{S} P_{R}$.

(a) Let ${ }_{R} M$ be F-projective and $P$ be a projector; then ${ }_{R} M$ is projective iff ${ }_{S} F(M)$ is projective and ${ }_{R} M$ is flat iff ${ }_{S} F(M)$ is flat.

(b) Let ${ }_{R} M$ be F-injective and $P$ be an injector; then ${ }_{R} M$ is injective iff ${ }_{S} F(M)$ is injective.

(c) Let ${ }_{S} N$ be G-injective and $P$ be a projector; then ${ }_{S} N$ is projective iff ${ }_{R} G(N)$ is projective and ${ }_{S} N$ is flat iff ${ }_{R} G(N)$ is flat.

(d) Let ${ }_{S} N$ be H-projective and $P$ be an injector; then ${ }_{S} N$ is injective iff ${ }_{R} H(N)$ is injective.

Proof. We prove (a); (b), (c), and (d) are similar.

For (a), if ${ }_{R} M$ is projective, then ${ }_{S} F(M)$ is projective since $P$ is a projector. Conversely, if ${ }_{S} F(M)$ is projective, ${ }_{R} G F(M)$ is projective so since ${ }_{R} M$ is $F$-projective, ${ }_{R} M$ is projective.

Similarly, if ${ }_{R} M$ is flat, then ${ }_{S} F(M)$ is flat since ${ }_{S} P$ is projective and hence flat. Conversely, if ${ }_{S} F(M)$ is flat, then ${ }_{R} G F(M)$ is flat since ${ }_{R} P^{*}$ is projective. Hence since ${ }_{R} M$ is $F$-projective, ${ }_{R} M$ must be flat.

We may generalize Proposition 5.1 and Theorem 5.2 to results on the homological dimensions of modules. Using induction and the long exact sequence of homology [16], it is easy to establish the following proposition.

5.3. Proposition. Let $R$ be $a$ ring and let $0 \rightarrow M_{n+1} \rightarrow M_{n} \rightarrow \cdots \rightarrow M_{1}$ $\rightarrow M_{0} \rightarrow 0$ be an exact sequence of left $R$-modules. For any integer $m \geqq 0$, we have:

(a) if $P d_{R}\left(M_{i}\right) \leqq m$ for $i=1,2, \ldots, n+1$, then $P d_{R}\left(M_{0}\right) \leqq n+m$,

(b) if $W d_{R}\left(M_{i}\right) \leqq m$ for $i=1,2, \ldots, n+1$, then $W d_{R}\left(M_{0}\right) \leqq n+m$,

(c) if $I d_{R}\left(M_{i}\right) \leqq m$ for $i=0,1, \ldots, n$, then $I d_{R}\left(M_{n+1}\right) \leqq n+m$.

5.4. Theorem. Let $R$ and $S$ be rings, and let ${ }_{S} P_{R}$ be a bimodule. Then for any left $R$-module $M$,

(a) if $P_{R}$ is flat, $P d_{S}\left(P \otimes_{R} M\right) \leqq P d_{S}\left({ }_{S} P\right)+P d_{R}(M)$ and $W d_{S}\left(P \otimes_{R} M\right) \leqq W d_{S}\left({ }_{S} P\right)$ $+W d_{R}(M)$.

(b) if $P_{R}$ is finitely generated and projective, $I d_{S}\left(P \otimes_{R} M\right) \leqq I d_{R}(M)+W d_{S}\left(P_{S}^{*}\right)$.

Proof. (a) is quite straightforward using (a) and (b) of Proposition 5.3. For (b), using Proposition 5.3(c), it will suffice to obtain a bound on $I_{S}\left(P \otimes_{R} Q\right)$ for an injective ${ }_{R} Q$. Since $P_{R}$ is finitely generated and projective, we have $P \otimes_{R} Q \cong P$ $\otimes_{R} \operatorname{Hom}_{R}(R, Q) \cong \operatorname{Hom}_{R}\left(P^{*}, Q\right)$; so using [6, Chapter VI, 5.1] together with the fact that ${ }_{R} Q$ is injective we have for any left $S$-module ${ }_{S} N$ that

$$
\operatorname{Ext}_{S}^{n}\left(N, P \otimes_{R} Q\right) \cong \operatorname{Ext}_{S}^{n}\left(N, \operatorname{Hom}_{R}\left(P^{*}, Q\right)\right) \cong \operatorname{Hom}_{R}\left(\operatorname{Tor}_{n}^{S}\left(P^{*}, N\right), Q\right) \text {. }
$$

Thus $I d_{S}\left(P \otimes_{R} Q\right) \leqq W d_{S}\left(P_{S}\right)$, and (b) follows. 
Note that if $P_{R}$ is finitely generated and projective and $S=\operatorname{End}_{R}\left(P_{R}\right)$, every $S$-module $N$ is of the form $P \otimes_{R} M$ for some ${ }_{R} M$ since ${ }_{S} S_{S} \cong{ }_{S} P \otimes_{R} P *$. It thus follows from Theorem 5.4 that

$$
\text { l.gl.dim. } S \leqq \text { l.gl.dim. } R+\min \left\{P d_{S}\left({ }_{S} P\right), W d_{S}\left(P_{S}^{*}\right)\right\}
$$

and

$$
\text { w.gl.dim. } S \leqq \text { w.gl.dim. } R+W d_{\mathrm{s}}\left({ }_{S} P\right),
$$

sharpening a result of Harada [11].

5.5. TheOREM. Let $(G, F, H)$ be a pairing of $R$ and $S$ with associated bimodule ${ }_{S} P_{R}$. If $P$ is both a projector and an injector, we have

(a) if ${ }_{R} M$ is $F$-projective, then $P d_{R}(M)=P d_{S}\left(F(M)\right.$ ) and $W d_{R}(M)=W d_{S}(F(M))$,

(b) if ${ }_{S} N$ is $G$-injective, then $P d_{S}(N)=P d_{R}(G(N))$ and $W d_{S}(N)=W d_{R}(G(N))$.

If in addition ${ }_{S} P$ is finitely generated, we have

(c) if ${ }_{R} M$ is F-injective, then $I d_{R}(M)=I d_{S}(F(M))$,

(d) if ${ }_{S} N$ is H-projective, then $\operatorname{Id}_{S}(N)=I d_{R}(H(N))$.

Proof. We prove (a) and (c); (b) and (d) are similar.

Since $P$ is both a projector and an injector, $P_{S}^{*}$ is flat and ${ }_{S} P$ is projective. Applying Theorem 5.3(a), we have $P d_{R}(M) \geqq P d_{S}(F(M)) \geqq P d_{R}(G F(M)) \geqq P d_{R}(M)$ since $M$ is $F$-projective. A similar argument is used for the weak dimensions, giving (a).

For (c), since ${ }_{S} P$ is finitely generated and projective, the functor $H$ has a right adjoint by Theorem 1.1, ( $\left.\mathrm{a}^{\prime}\right)$ and $\left(\mathrm{b}^{\prime}\right)$. We may apply Theorem 5.3(b) to the functor $H$, obtaining the inequalities $I d_{R}(M) \geqq I d_{S}(F(M)) \geqq I d_{R}(H F(M)) \geqq I d_{R}(M)$ since $M$ is $F$-injective.

5.6. Corollary. Let $(G, F, H)$ be a pairing of $R$ and $S$ with an associated bimodule ${ }_{S} P_{R}$ which is both a projector and an injector.

(a) If all ${ }_{R} M$ are $F$-projective and all ${ }_{S} N$ are $G$-injective, then $1 . g l \cdot \operatorname{dim} R=1 . g 1 . \operatorname{dim} S$ and w.gl.dim $R=$ w.gl.dim $S$.

If in addition ${ }_{S} P$ is finitely generated,

(b) if all ${ }_{R} M$ are F-injective and all ${ }_{S} N$ are $H$-projective, then $1 . g 1 . \operatorname{dim} R=$ 1.gl.dim $S$.

Semisimple, left hereditary, and von Neumann regular rings are characterized by their global dimensions, so that if $R$ and $S$ are paired in a way satisfying the hypotheses of Corollary 5.5 (a), and if one of $R$ or $S$ is semisimple, left hereditary, or von Neumann regular, the other must be also.

In $[3, \S 5]$, Bass defined the finistic dimensions

$$
\begin{aligned}
\operatorname{LFPD}(R) & =\sup \left\{P d_{R}(M) \mid P d_{R}(M)<\infty\right\}, \\
L F W D(R) & =\sup \left\{W d_{R}(M) \mid W d_{R}(M)<\infty\right\}, \\
L F I D(R) & =\sup \left\{I d_{R}(M) \mid I d_{R}(M)<\infty\right\}, \\
L f P D(R) & =\sup \left\{P d_{R}(M) \mid{ }_{R} M \text { is finitely generated and } P d_{R}(M)<\infty\right\}
\end{aligned}
$$


Theorem 5.4 makes it clear that if $R$ and $S$ are paired in a way satisfying the hypotheses of Corollary 5.5(a), then $L F P D(R)=L F P D(S)$ and $L F W D(R)=L F W D(S)$, while if the pairing satisfies the hypotheses of Corollary 5.5(b), $L F I D(R)=L F I D(S)$. To consider the finistic dimension $L f P D$, we need the following proposition.

5.7. Proposition. Let $R$ and $S$ be rings and let ${ }_{S} U_{R}$ be a bimodule. Then ${ }_{S} U \otimes_{R} M$ is finitely generated for all finitely generated ${ }_{R} M$ iff ${ }_{S} U$ is finitely generated.

Proof. Letting ${ }_{R} M={ }_{R} R$, it is clear that it is necessary that ${ }_{S} U$ be finitely generated.

Conversely, suppose that ${ }_{S} U$ is finitely generated and let ${ }_{R} M$ be finitely generated. Then there is an epimorphism $\oplus \sum_{n} R \rightarrow{ }_{R} M \rightarrow 0$, so that the sequence $\oplus \sum_{n} S$ $\otimes_{R} R \rightarrow{ }_{S} U \otimes_{R} M \rightarrow 0$ is exact. Since ${ }_{S} U$ is finitely generated, ${ }_{S} U \otimes_{R} M$ must be finitely generated.

Thus if a pairing of $R$ and $S$ satisfies the hypotheses of corollary 5.5(a) with the associated module ${ }_{S} P$ finitely generated, we have $L f P D(R)=L f P D(S)$.

Cartan and Eilenberg [6, p. 15] show that $R$ is left semihereditary iff every finitely generated submodule of a projective left $R$-module is projective.

5.8. Proposition. Let $(G, F, H)$ be a pairing of $R$ and $S$ with an associated bimodule ${ }_{S} P_{R}$ which is both a projector and an injector. Suppose that all ${ }_{R} M$ are $F$-projective and that all ${ }_{S} N$ are $G$-injective. If $R$ is left semihereditary, then $S$ is left semihereditary. The converse holds if ${ }_{S} P$ is finitely generated.

Proof. Let $R$ be left semihereditary, and let ${ }_{S} K$ be a finitely generated submodule of the projective module ${ }_{S} N$. Since $P_{S}^{*}$ is flat and ${ }_{R} P^{*}$ is finitely generated and projective, $G(K)$ is isomorphic to a finitely generated submodule of the projective module $G(N)$. Thus $G(K)$ is projective, whence by Theorem 5.2(c), $K$ is projective. So $S$ is left semihereditary.

The converse is a duplicate of the above argument, noting that we must have $F$ preserve finitely generated modules; i.e., ${ }_{s} P$ must be finitely generated.

We now consider left self-injective rings.

5.9. Proposition. Let $(G, F, H)$ be a pairing of $R$ and $S$ whose associated bimodule ${ }_{S} P_{R}$ is an injector, and suppose that ${ }_{S} S$ is $G$-injective. Then if $R$ is left self-injective, $S$ is left self-injective.

Proof. The module ${ }_{R} P^{*} \cong{ }_{R} G(S)$ is finitely generated and projective, so that ${ }_{R} G(S)$ is injective. Thus ${ }_{S} F G(S)$ is injective, so since ${ }_{S} S$ is $G$-injective, ${ }_{S} S$ is isomorphic to a direct summand of ${ }_{S} F G(S)$ and is thus injective.

Now let us consider rings with chain conditions on left or right ideals. Our main result in this direction is based upon the faithfully exact functors of Ishikawa [15]. A functor $T:{ }_{R} \mathscr{M} \rightarrow{ }_{S} \mathscr{M}$ is faithfully exact in case a sequence

$$
A \stackrel{f}{\longrightarrow} B \stackrel{g}{\longrightarrow} C
$$


of $R$-modules is exact iff the sequence

$$
T(A) \stackrel{T(f)}{\longrightarrow} T(B) \stackrel{T(g)}{\longrightarrow} T(C)
$$

of $S$-modules is exact. Ishikawa characterized these as follows.

5.10. TheOREM [15, THEOREM 1.1]. Let $T:{ }_{R} \mathscr{M} \rightarrow{ }_{S} \mathscr{M}$ be an exact functor. Then the following are equivalent:

(a) $T$ is faithfully exact,

(b) $T(f) \neq 0$ for any nonzero $R$-homomorphism $f$,

(c) $T(X) \neq 0$ for any nonzero $R$-module $X$.

A right $R$-module $P_{R}$ is called faithfully flat in case the functor $P \otimes_{R}$ ( ) is faithfully exact. Ishikawa [15, Propositions $2.3,2.4$ ] showed that if $P_{R}$ is finitely generated and projective, then $P_{R}$ is faithfully flat iff $P_{R}$ is a progenerator. It follows from 3.4(c) that if ${ }_{S} P_{R}$ is a bimodule associated with a strongly separable pairing, $P_{R}$ is faithfully flat. The following theorem thus applies to strongly separable pairings.

5.11. Theorem. Let ${ }_{S} P_{R}$ be a bimodule with $P_{R}$ faithfully flat. Let $M \in_{R} \mathscr{M}$, and for ${ }_{R} M^{\prime} \subseteq_{R} M$, let $\varphi\left(M^{\prime}\right)$ be the natural image of $P \otimes_{R} M^{\prime}$ in $P \otimes_{R} M$. Then $\varphi$ defines a one-to-one order-preserving map of the lattice of submodules of $M$ into the lattice of submodules of $P \otimes_{R} M$.

Proof. We first claim that if $M^{\prime}$ is a proper submodule of $M, \varphi\left(M^{\prime}\right)$ is a proper submodule of $P \otimes_{R} M$. For if $0 \rightarrow M^{\prime} \stackrel{i}{\rightarrow} M \rightarrow M / M^{\prime} \rightarrow 0$ is exact with $M / M^{\prime}$ $\neq 0,0 \rightarrow P \otimes_{R} M^{\prime} \stackrel{1 \otimes 1}{\longrightarrow} P \otimes_{R} M \rightarrow P \otimes_{R}\left(M / M^{\prime}\right) \rightarrow 0$ is exact with $P \otimes_{R}\left(M / M^{\prime}\right)$ $\neq 0$. Thus $\left(P \otimes_{R} M\right) / \varphi\left(M^{\prime}\right) \cong P \otimes_{R}\left(M / M^{\prime}\right)$ is nonzero, so $\varphi\left(M^{\prime}\right)$ is a proper submodule of $P \otimes_{R} M$.

Now $\varphi$ is clearly order-preserving; we must show that $\varphi$ is one-to-one. Let $M^{\prime}$, $M^{\prime \prime}$ be submodules of $M$ with $M^{\prime}=M^{\prime \prime}$. Then $M^{\prime} \cap M^{\prime \prime}$ is a proper submodule of $M^{\prime}$, so that $\varphi\left(M^{\prime} \cap M^{\prime \prime}\right)$ is a proper submodule of $\varphi\left(M^{\prime}\right)$. But by [5, Lemma 7, p. 32], $\varphi\left(M^{\prime} \cap M^{\prime \prime}\right)=\varphi\left(M^{\prime}\right) \cap \varphi\left(M^{\prime \prime}\right)$; so $\varphi\left(M^{\prime}\right) \cap \varphi\left(M^{\prime \prime}\right)$ is a proper submodule of $\varphi\left(M^{\prime}\right)$. Thus $\varphi\left(M^{\prime}\right) \neq \varphi\left(M^{\prime \prime}\right)$ and $\varphi$ is one-to-one.

5.12. Corollary. Let ${ }_{S} P_{R}$ be a bimodule with $P_{R}$ faithfully flat. For an $R$-module $M$, if ${ }_{S} P \otimes_{R} M$ is artinian (respectively noetherian), so is $M$. In particular, if ${ }_{S} P$ is finitely generated and $S$ is left artinian (respectively left noetherian), $R$ is left artinian (respectively left noetherian).

Proof. The first statement is straightforward. The second follows since $R$ is left artinian (left noetherian) iff every finitely generated ${ }_{R} M$ is artinian (noetherian).

In particular, if $R$ and $S$ are strongly separably paired, and if either of $R$ or $S$ has a chain condition on left ideals, so must the other. However, unlike rings which have equivalent categories of modules, there need not be an isomorphism between the lattices of two-sided ideals of $R$ and $S$. For example, let $K$ be a field and $G$ be a finite group such that the characteristic of $K$ is prime to $|G|$. Then $K G$ is a strongly separable extension of $K$, but in general $K G$ is not simple. 
We now examine some special types of rings. For these, we shall assume that $R$ and $S$ are strongly separably paired, although the results may hold under somewhat weaker hypotheses.

5.13. Proposition. Let $R$ and $S$ be strongly separably paired. Then $R$ is $Q F$ iff $S$ is $Q F$.

Proof. Recall that $R$ is QF iff $R$ is left noetherian and left self-injective (see [23]). The result then follows by Corollary 5.11 and Proposition 5.8.

Cogenerator rings (see [23]) generalize QF rings. A ring $R$ is a cogenerator ring in case ${ }_{R} R$ is a cogenerator; i.e., in case for any ${ }_{R} M$ there is a monomorphism $0 \rightarrow{ }_{R} M \rightarrow \prod_{A R} R$ for some index set $A$.

5.14. Proposition. Let $R$ and $S$ be strongly separably paired. Then $R$ is a cogenerator ring iff $S$ is a cogenerator ring.

Proof. Let $(G, F, H)$ be a strongly separable pairing of $R$ and $S$ with associated bimodule ${ }_{S} P_{R}$. For any ${ }_{R} M$, we have $P \otimes_{R} M \cong \operatorname{Hom}_{R}\left(P^{*}, M\right)$, so since

$$
\operatorname{Hom}_{R}\left(P^{*}, \prod_{A} M_{\alpha}\right) \cong \prod_{A} \operatorname{Hom}_{R}\left(P^{*}, M_{\alpha}\right)
$$

we have $P \otimes_{R} \prod_{A} M_{\alpha} \cong \prod_{A} P \otimes_{R} M_{\alpha}$.

Since ${ }_{R} R$ is a cogenerator, for any ${ }_{S} N$ there is a monomorphism $0 \rightarrow H(N)$ $\rightarrow \prod_{A R} R$ for some $A$. Then $0 \rightarrow F H(N) \rightarrow \prod_{A S} P$ is a monomorphism, so since ${ }_{S} N$ is $H$-projective, there is a monomorphism $0 \rightarrow N \rightarrow \prod_{A} P$. Thus ${ }_{S} P$ is a cogenerator. But ${ }_{s} P$ is finitely generated and projective, so ${ }_{S} S$ is a cogenerator.

The converse is by symmetry.

As another generalization of QF rings we have the left QF-3 rings of [9]. A ring $R$ is left QF-3 in case $R$ is left artinian and the injective hull $E(R)$ of ${ }_{R} R$ is projective.

5.15. Proposition. Let $R$ and $S$ be strongly separably paired. Then $R$ is left $Q F-3$ iff $S$ is left $Q F-3$.

Proof. Let $R$ be left QF-3. Then $S$ is left artinian by Corollary 5.11, so we must show that $E\left({ }_{S} S\right)$ is projective.

Let $(G, F, H)$ be a strongly separable pairing of $R$ and $S$ with associated bimodule ${ }_{S} P_{R}$. Now $E(R)$ is projective, so since ${ }_{R} P^{*}$ is finitely generated and projective, $E\left({ }_{R} P^{*}\right)$ is projective. Then $F\left(E\left({ }_{R} P^{*}\right)\right)$ is projective. But $F\left(E\left({ }_{R} P^{*}\right)\right)$ contains a submodule isomorphic to $F\left(P^{*}\right)$, so $E\left({ }_{s} F\left(P^{*}\right)\right)$ must be projective. Finally, since ${ }_{S} S$ is isomorphic to a direct summand of $F\left(P^{*}\right) \cong F G(S), E\left({ }_{S} S\right)$ must be projective. Thus $S$ is left QF-3.

Again, the converse is by symmetry.

Finally, left perfect rings were characterized by Bass [3, p. 468] as those rings for which every direct limit of projective modules is projective. But Govorov [11] proved that every flat module is a direct limit of projective modules, so $R$ is left perfect iff every flat $R$-module is projective. The following proposition is then clear by Theorem 5.2. 
5.16. Proposition. Let $R$ and $S$ be strongly separably paired. Then $R$ is left perfect iff $S$ is left perfect.

\section{BIBLIOGRAPHY}

1. F. W. Anderson, Endomorphism rings of projective modules, Math. Z. 111 (1969), 322-332.

2. M. Auslander and $\mathrm{O}$. Goldman, The Brauer group of a commutative ring, Trans. Amer. Math. Soc. 97 (1960), 367-409. MR 22 \#12130.

3. H. Bass, Finitistic dimension and a homological generalization of semi-primary rings, Trans. Amer. Math. Soc. 95 (1960), 466-488. MR 28 \#1212.

4. - The Morita theorems, Lecture Notes, Univ. of Oregon, Eugene, 1962.

5. N. Bourbaki, Algèbre commutative. Chapitres I, II, Actualités Sci. Indust., no. 1290, Hermann, Paris, 1961. MR 36 \#146.

6. H. Cartan and S. Eilenberg, Homological algebra, Princeton Univ. Press, Princeton, N. J., 1956. MR 17, 1040.

7. C. W. Curtis and I. Reiner, Representation theory of finite groups and associative algebras, Pure and Appl. Math., vol. 11, Interscience, New York, 1962. MR 26 \#2519.

8. K. Fuller, The structure of QF-3 rings, Trans. Amer. Math. Soc. 134 (1968), 343-354. MR 37 \#2810.

9. N. S. Gopalakrishnan, et al., A note on the dimension of modules and algebras, J. Indian Math. Soc. 21 (1957), 185-192. MR 25 \#109.

10. V. E. Govorov, Rings over which flat modules are free, Dokl. Akad. Nauk SSSR 144 (1962), 965-967 = Soviet Math. Dokl. 3 (1962), 836-838. MR 25 \#3076.

11. M. Harada, Note on the dimension of modules and algebras, J. Inst. Polytech. Osaka City Univ. Ser. A 7 (1956), 17-27. MR 18, 375.

12. K. Hirata, On relative homological algebra of Frobenius extensions, Nagoya Math. J. 15 (1959), 17-28. MR 21 \#7242.

13. K. Hirata and K. Sugano. On semisimple extensions and separable extensions over noncommutative rings, J. Math. Soc. Japan 18 (1966), 360-373. MR 34 \#208.

14. G. Hochschild, Relative homological algebra, Trans. Amer. Math. Soc. 82 (1956), 246-269. MR 18, 278.

15. T. Ishikawa, Faithfully exact functors and their applications to projective modules and injective modules, Nagoya Math. J. 24 (1964), 29-42. MR 30 \#131.

16. J. P. Jans, Rings and homology, Holt, New York, 1964. MR 29 \#1243.

17. D. M. Kan, Adjoint functors, Trans. Amer. Math. Soc. 87 (1958), 294-329. MR 24 \#A1301.

18. F. Kasch, Grundlagen einer Theorie der Frobeniuserweiterungen, Math. Ann. 127 (1954), 453-474. MR 16, 7.

19. J. Lambek, Lectures on rings and modules, Blaisdell, Waltham, Mass., 1966. MR 34 \#5857.

20. K. Morita, Duality for modules and its applications to the theory of rings with minimum conditions, Sci. Rep. Tokyo Kyoiku Daigaku Sect. A 6 (1958), 83-142. MR 20 \#3183.

21. - Adjoint pairs of functors and Frobenius extensions, Sci. Rep. Tokyo Kyoiku Daigaku Sect. A 9 (1965), 40-71. MR 32 \#7597.

22. B. Müller, Quasi-Frobenius-Erweiterungen, Math. Z. 85 (1964), 345-368. MR 32 \#126.

23. —_, Quasi-Frobenius-Erweiterungen. II, Math. Z. 88 (1965), 380-409. MR 32 \#5696.

24. B. Osofsky, A generalization of quasi-Frobenius rings, J. Algebra 4 (1966), 373-387. MR 34 \#4305.

UNIVERSITY OF OREGON, EUGENE, OREGON 97403 